\title{
Efeitos de longo prazo do estresse neonatal com lipopolissacarídeo em ratos
}

\author{
Long-term effects of neonatal stress using lipopolysaccharide in rats
}

\author{
Adroaldo Lunardelli ${ }^{1}$, Carolina Luft², Leonardo Pedrazza1, \\ Márcio Vinícius Fagundes Donadio ${ }^{3}$
}

\begin{abstract}
RESUMO
Introdução: Diversos modelos experimentais têm sido utilizados para demonstrar que intervenções no início da vida podem gerar alterações permanentes que perduram ao longo da vida. A administração de lipopolissacarídeo (LPS) no período neonatal gera um estímulo imunológico estressante capaz de alterar muitas respostas fisiológicas ao estresse na vida adulta.

Objetivo: Revisar a literatura acerca das influências, em longo prazo, que a administração de LPS no período neonatal pode gerar na vida adulta em modelos experimentais.

Materiais e Métodos: O presente estudo consiste em uma revisão integrativa da literatura com base na busca de artigos científicos disponíveis nas bases de dados Medline/PubMed e Science Direct, utilizando os descritores neonatal programming, neonatal stress, neonatal LPS e neonatal lipopolysaccharide. Foram incluídas publicações cuja temática abordasse os resultados da utilização de LPS como estressor neonatal em protocolos experimentais, sem limite de data. Resultados: Foram selecionados 15 artigos que mostram modelos experimentais em que a injeção de LPS em ratos neonatos causa modificações funcionais da resposta do eixo hipotálamo-hipófise-adrenal (HPA) quando adultos, incluindo elevação nos níveis plasmáticos de corticosterona. Ainda, há diminuição das concentrações circulantes de citocinas pró-inflamatórias, hiperalgesia, aumento na sensibilidade ao estresse e aumento do comportamento de ansiedade e depressão.
\end{abstract}

Conclusão: Os resultados demonstram que a administração neonatal de LPS consiste em um modelo experimental efetivo de programming, provocando uma série de alterações imunológicas e comportamentais na vida adulta.

Palavras-chave: animais recém-nascidos; inflamação; modelos animais; estresse fisiológico.

\begin{abstract}
Introduction: Several experimental models have been used to demonstrate that interventions early in life can lead to permanent changes that persist throughout life. In this context, the administration of lipopolysaccharide (LPS) in the neonatal period generates a stressful immune stimulation, which is able to change many physiological responses to stress in adulthood.

Objective: To review the literature about the long-term influences in the adult life of the LPS administration during the neonatal period in experimental models.

Materials and Methods: This study consists of an integrative literature review based on the search of scientific articles available in Medline/Pubmed and Science Direct databases, using the following keywords: neonatal programming, neonatal stress, neonatal LPS and neonatal lipopolysaccharide. We have included manuscripts whose content included the use of LPS as a neonatal stressor in experimental protocols, without limits of date.

Results: We selected 15 articles demonstrating that experimental models injecting LPS in neonatal rats results in functional modifications of the hypothalamic-pituitary-adrenal (HPA) axis as adults, including increased levels of plasma corticosterone. Indeed, there are decreased inflammatory cytokine levels, hyperalgesia, increased sensitivity to stress and increased anxiety and depression behaviors.

Conclusion: The results demonstrate that neonatal LPS administration consists of an effective experimental model of programming, leading to a series of immunological and behavioral changes in adulthood.
\end{abstract}

Keywords: newborn animals; inflammation; animal models; physiological stress.

${ }^{1}$ Farmacêutico. Mestre em Biologia Celular e Molecular. Laboratório de Pesquisa em Biofísica Celular e Inflamação, Pontifícia Universidade Católica do Rio Grande do Sul (PUCRS), Brasil.

${ }^{2}$ Acadêmica em Ciências Biológicas. Laboratório de Pesquisa em Biofísica Celular e Inflamação, PUCRS, Brasil.

${ }^{3}$ Fisioterapeuta. Doutor em Fisiologia pela Universidade Federal do Rio Grande do Sul. Pesquisador do Centro Infant, Instituto de Pesquisas Biomédicas, PUCRS. Professor do Programa de Pós-Graduação em Pediatria e Saúde da Criança e da Faculdade de Enfermagem, Nutrição e Fisioterapia, PUCRS, Brasil. 


\section{INTRODUÇÃO}

Diversos modelos experimentais têm sido utilizados para demonstrar que intervenções no início da vida, sejam elas pré ou neonatais, podem gerar alterações permanentes que perduram ao longo da vida. $O$ conceito de programming tem sido desenvolvido para explicar o processo pelo qual um organismo se adapta aos eventos ambientais através da geração de alterações estáveis no fenótipo. Isto geralmente ocorre em períodos iniciais de desenvolvimento, fazendo com que a exposição a um ambiente adverso possa perturbar o processo de proliferação e diferenciação celular e tenha efeitos duradouros sobre a saúde do indivíduo ${ }^{1}$.

O estresse imune pré-natal induzido por lipopolissacarídeo (LPS) ou citocinas, aumenta a ingestão de comida em ratos e leva à obesidade e a distúrbios associados à síndrome metabólica, como o aumento de leptina sérica e resistência à insulina no adulto $^{2}$. Também, modelos como a manipulação no período neonatal têm sido amplamente estudados com a descrição de inúmeras alterações, incluindo diminuição do medo ao explorar ambientes novos ${ }^{3}$, resposta menos acentuada na secreção de glicocorticoides ${ }^{4,5}$, diminuição da função reprodutiva ${ }^{1,6,7}$, diminuição do número de células no locus coeruleus ${ }^{8}$, redução da densidade de receptores de angiotensina II na área pré-óptica medial e no núcleo paraventricular ${ }^{9}$, além de importante redução na função renal e nos mecanismos de regulação do equilíbrio hidroeletrolítico ${ }^{10}$. De qualquer forma, independente do modelo utilizado, parece bem estabelecido que intervenções nestes períodos importantes provoquem este efeito de programming, ou seja, uma "marca" no desenvolvimento de diferentes sistemas que permanece ao longo da vida.

Este parece também ser o caso de modelos que reproduzem situações de inflamação e/ou infecção durante o período neonatal e alteram a responsividade de importantes sistemas na vida adulta ${ }^{11}$. O período neonatal é um estágio muito importante no desenvolvimento neurológico ${ }^{12}$, pois se caracteriza por grande plasticidade e capacidade de reorganização ${ }^{13}$. A administração de LPS no período neonatal gera um estímulo imunológico estressante ${ }^{14}$ capaz de estimular a expressão de citocinas e causar alterações cerebrais em certas regiões do hipocampo de ratos adultos ${ }^{15}$, além de ativar o eixo hipotálamo-pituitária-adrenal (HPA) em neonatos ${ }^{16}$. Já é sabido que a injeção de LPS em ratos neonatos causa elevações nos níveis plasmáticos de corticosterona e aumenta a sensibilidade do eixo HPA quando adultos, o que altera muitas respostas fisiológicas ao estresse na vida adulta ${ }^{17}$. Além disso, a administração de LPS neonatal aliado a uma exposição estressante quando adulto resulta em aumento significativo no comportamento de ansiedade ${ }^{16}$.

À parte das evidências em modelos experimentais, nos seres humanos, uma experiência adversa no início da vida, como negligência emocional, pode levar a um aumento da probabilidade do indivíduo desenvolver ansiedade, depressão, doença crônica ou mesmo vício em drogas na vida futura ${ }^{18}$. Achados semelhantes foram demonstrados em modelos de primatas com privação parental ou social durante o período neonatal, produzindo adultos socialmente desajustados, tímidos, deprimidos e com alterações na função neuroimune e no eixo $\mathrm{HPA}^{18}$. Dessa forma, o período neonatal constitui-se em uma fase importante do neurodesenvolvimento para a compreensão da etiologia e da trajetória do desenvolvimento de desordens neuropsiquiátricas na vida adulta, como a ansiedade e a depressão ${ }^{12}$.

Assim, o entendimento das repercussões e mecanismos pelos quais variações no período neonatal podem gerar alterações estáveis que perduram ao longo da vida consiste em tema atual e de grande relevância. A alta prevalência de crianças que passam por situações adversas no período neonatal, incluindo processos inflamatórios, justifica a preocupação em conhecer os efeitos dessas intervenções sobre a resposta a situações adversas na vida adulta. Com base nisso, este estudo tem como objetivo revisar a literatura acerca das influências, em longo prazo, que a administração de LPS no período neonatal pode gerar na vida adulta em modelos experimentais.

\section{MATERIAIS E MÉTODOS}

O presente estudo consiste em uma revisão 
integrativa da literatura com base na busca de artigos científicos disponíveis em diferentes bases de dados. A busca eletrônica nas bases de dados Medline/PubMed e Science Direct, que fornecem informação técnicocientífica de reconhecida qualidade, foi realizada no mês de junho de 2013 utilizando os descritores/unitermos, em língua inglesa, neonatal programming, neonatal stress, neonatal LPS e neonatal lipopolysaccharide. Adotou-se como critérios de inclusão as publicações sem limite de data - cuja temática abordasse os resultados da utilização de LPS como estressor neonatal em protocolos experimentais. Primeiramente, foram analisados títulos e resumos dos resultados das buscas, sendo aqueles estudos que contemplassem a temática proposta selecionados, incluídos e lidos de forma minuciosa. Foram excluídos da seleção estudos realizados em seres humanos e revisões de literatura. $A$ análise minuciosa seguiu referencial teórico, incluindo a leitura exploratória, seletiva, analítica e interpretativa do material empírico ${ }^{19}$.

\section{RESULTADOS E DISCUSSÃO}

Através das buscas realizadas e de acordo com os critérios descritos anteriormente, foram selecionados e incluídos 15 artigos nesta revisão. Estes são tabulados na tabela 1, que apresenta os trabalhos que fazem uso de LPS como indutor de programming neonatal.

A endotoxina LPS é um componente pirogênico imunologicamente ativo da parede celular de bactérias gram negativas endêmica à maioria das espécies de mamíferos. Os vertebrados têm receptores específicos de LPS na superfície de macrófagos, sendo que a administração de LPS imita a resposta comportamental, neuroquímica e neuroendócrina produzida pelo sistema imune em resposta à infecção ${ }^{20}$. Um único desafio com LPS no período neonatal é capaz de gerar alterações de longo prazo (que permanecem até a vida adulta) na resposta de sistemas fisiológicos ${ }^{21}$. Modelos experimentais de indução de programming tem utilizado uma aplicação de LPS no décimo ${ }^{2,17,21}$ ou décimo quarto $22-24$ dia de vida. No entanto, outros estudos fazem duas aplicações, nos dias 3 e $5^{16,25-31}$ ou nos dias 7 e $9^{32}$ após o nascimento. De maneira geral, os trabalhos fazem uso de ratos Wistar, Long-Evans, Fischer-344, Lister-hooded ou Sprague-Dawley.

Uma infecção bacteriana, tal como a mimetizada pela aplicação de LPS, manifesta a resposta central a um desafio imunológico. Patógenos (como o LPS) se ligam a receptores toll-like (por exemplo, o receptor de toll-like 4, TLR4) em monócitos, macrófagos e adipócitos. Esta ativação inicia uma cascata que contribui para a iniciação da resposta febril e liberação local de prostaglandina E2 (PGE2) a partir do fígado, que atua sobre o vago gerando uma sinalização para o sistema nervoso central e causando elevação inicial de glicocorticoides através do eixo HPA. Posteriormente, há uma série de eventos que culminam com a fosforilação do inibidor IKB, liberando o fator nuclear NFKB que é translocado para o núcleo, onde inicia a transcrição de citocinas pró-inflamatórias, tais como a interleucina $1 \beta$ (IL-1 $\beta$ ), interleucina 6 (IL-6) e fator de necrose tumoral $\alpha$ (TNF- $\alpha)$, bem como as citocinas antiinflamatórias como o antagonista do receptor de IL-1 e interleucina 10 (IL-10). As citocinas são liberadas para a corrente sanguínea e estimulam a enzima ciclooxigenase 2 (COX-2), que regula a conversão de ácido araquidônico para prostaglandina E2 (PGE2). Esta sequência de eventos atua sobre o sistema nervo central induzindo uma série de respostas fisiológicas que culminam com a produção de febre e ativação mais pronunciada do eixo HPA na luta contra o patógeno invasor ${ }^{33}$.

Em relação ao estudo dos possíveis efeitos cumulativos de eventos perinatais, parece haver necessidade de uma abordagem mais integradora. Neste sentido, a utilização de estratégias como um "duplo estímulo" fornece uma plataforma a partir da qual se pode investigar a inter-relação dinâmica entre múltiplos mecanismos, postulando que um primeiro estímulo (genético ou ambiental) durante períodos críticos do desenvolvimento atrapalha a ontogenia dos sistemas neurais, que estabelece uma susceptibilidade a um segundo estímulo na vida adulta $^{16}$. Animais adultos submetidos à administração de LPS têm concentrações circulantes das citocinas próinflamatórias, IL-1 $\beta$, IL-6 e TNF- $\alpha$, significativamente atenuadas quando tratados com LPS no período 
TABELA 1 - Resumo dos principais estudos que fazem uso de LPS como indutor de programming experimental neonatal.

\begin{tabular}{|c|c|c|c|c|c|}
\hline Autor & Ano & Animal & $\begin{array}{l}\text { Idade (dias) } \\
\text { aplicação LPS }\end{array}$ & $\begin{array}{l}\text { LPS (tipo e } \\
\text { concentração) }\end{array}$ & Principais achados \\
\hline Iwasa et al. ${ }^{2}$ & 2010 & $\begin{array}{l}\text { Sprague- } \\
\text { Dawley }\end{array}$ & 10 & $100 \mu \mathrm{g} / \mathrm{Kg}$ & $\begin{array}{l}\text { Aumento no peso corporal, aumento na } \\
\text { injestão de alimento, diminuição de citocinas } \\
\text { no hipotálamo e aumento de leptina sérica. }\end{array}$ \\
\hline Iwasa et al. ${ }^{17}$ & 2009 & $\begin{array}{l}\text { Sprague- } \\
\text { Dawley }\end{array}$ & 10 & $100 \mu \mathrm{g} / \mathrm{Kg}$ & $\begin{array}{l}\text { Prevenção da supressão do hormônio } \\
\text { luteinizante e atenuação do aumento de } \\
\text { citocinas hipotalâmicas. }\end{array}$ \\
\hline Iwasa et al. ${ }^{21}$ & 2009 & $\begin{array}{l}\text { Sprague- } \\
\text { Dawley }\end{array}$ & 10 & $100 \mu \mathrm{g} / \mathrm{Kg}$ & $\begin{array}{l}\text { Prolongamento do ciclo estral, aumento na } \\
\text { expressão hipotalâmica de urocortina } 2 \text { e } \\
\text { receptores de corticotropina. }\end{array}$ \\
\hline Spencer et al. ${ }^{22}$ & 2010 & $\begin{array}{l}\text { Sprague- } \\
\text { Dawley }\end{array}$ & 14 & $\begin{array}{l}\text { Escherichia coli } \\
100 \mu \mathrm{g} / \mathrm{Kg}\end{array}$ & $\begin{array}{l}\text { Redução da hipotermia e aumento da } \\
\text { hipertermia. }\end{array}$ \\
\hline Kentner et al. ${ }^{23}$ & 2010 & $\begin{array}{l}\text { Sprague- } \\
\text { Dawley }\end{array}$ & 14 & $\begin{array}{l}\text { Escherichia coli } \\
100 \mu \mathrm{g} / \mathrm{Kg}\end{array}$ & $\begin{array}{l}\text { Diminuição de interleucina-6 sérica e da } \\
\text { expressão hipotalâmica de COX-2. }\end{array}$ \\
\hline Boissé et al. ${ }^{24}$ & 2005 & $\begin{array}{l}\text { Sprague- } \\
\text { Dawley }\end{array}$ & 14 & $\begin{array}{l}\text { Escherichia coli } \\
100 \mu \mathrm{g} / \mathrm{Kg}\end{array}$ & $\begin{array}{c}\text { Redução da expressão de COX-2 da medula } \\
\text { espinhal. }\end{array}$ \\
\hline Walker et al. ${ }^{16}$ & 2009 & Wistar & 3 e 5 & $\begin{array}{l}\text { Salmonella } \\
\text { enterica } 50 \\
\quad \mu \mathrm{g} / \mathrm{Kg}\end{array}$ & $\begin{array}{l}\text { Aumento no comportamento de ansiedade e } \\
\text { de corticosterona. }\end{array}$ \\
\hline Knox et al. ${ }^{25}$ & 2009 & $\begin{array}{l}\text { Sprague- } \\
\text { Dawley }\end{array}$ & 3 e 5 & $\begin{array}{l}\text { Escherichia coli } \\
\qquad 50 \mu \mathrm{g} / \mathrm{Kg}\end{array}$ & Diminuição da kisspeptina hipotalâmica. \\
\hline Tenk et al. ${ }^{26}$ & 2007 & $\begin{array}{l}\text { Long- } \\
\text { Evans }\end{array}$ & 3 e 5 & $\begin{array}{l}\text { Escherichia coli } \\
\qquad 50 \mathrm{\mu g} / \mathrm{Kg}\end{array}$ & $\begin{array}{l}\text { Potencialização da ação da atividade } \\
\text { locomotora do quimpirole em fêmeas. }\end{array}$ \\
\hline Li et al. ${ }^{27}$ & 2007 & $\begin{array}{l}\text { Sprague- } \\
\text { Dawley }\end{array}$ & 3 e 5 & $\begin{array}{l}\text { Escherichia coli } \\
\qquad 50 \mu \mathrm{g} / \mathrm{Kg}\end{array}$ & $\begin{array}{l}\text { Aumento do hormônio luteinizante sérico e do } \\
\text { receptor de corticotropina pré-óptica. }\end{array}$ \\
\hline Shanks et al. ${ }^{28}$ & 2000 & $\begin{array}{l}\text { Sprague- } \\
\text { Dawley }\end{array}$ & 3 e 5 & $\begin{array}{l}\text { Salmonella } \\
\text { enteritidis } 50 \\
\mu \mathrm{g} / \mathrm{Kg}\end{array}$ & $\begin{array}{l}\text { Aumento de corticosterona plasmática, } \\
\text { diminuição de corticotropina e aumento de } \\
\text { arginina-vasopressina em núcleo } \\
\text { paraventricular. }\end{array}$ \\
\hline Walker et al. ${ }^{29}$ & 2010 & Wistar & 3 e 5 & $\begin{array}{l}\text { Salmonella } \\
\text { enterica } 50 \\
\quad \mu \mathrm{g} / \mathrm{Kg}\end{array}$ & $\begin{array}{l}\text { Aumento da corticosterona plasmática, de } \\
\text { interleucina-1 e do fator de necrose tumoral- } \alpha \\
\text { no hipocampo quando estresse por } \\
\text { contenção. }\end{array}$ \\
\hline Sominsky et al. ${ }^{30}$ & 2013 & Wistar & 3 e 5 & $\begin{array}{l}\text { Salmonella } \\
\text { enterica } 50 \\
\quad \mu \mathrm{g} / \mathrm{Kg}\end{array}$ & $\begin{array}{l}\text { Aumento no comportamento de ansiedade, } \\
\text { aumento da corticosterona. Aumento do } \\
\text { receptor de glicocorticoide provavelmente por } \\
\text { um estado de resistência ao glicocorticoide. }\end{array}$ \\
\hline Walker et al. ${ }^{31}$ & 2006 & $\begin{array}{l}\text { Fischer- } \\
344\end{array}$ & 3 e 5 & $\begin{array}{c}\text { Salmonella } \\
\text { enteritidis } 50 \\
\mu \mathrm{g} / \mathrm{Kg}\end{array}$ & Atenuação da resposta febril. \\
\hline Jenkins et al. ${ }^{32}$ & 2009 & $\begin{array}{l}\text { Lister- } \\
\text { hooded }\end{array}$ & 7 e 9 & $\begin{array}{l}\text { Escherichia coli } \\
500 \mu \mathrm{g} / \mathrm{Kg}\end{array}$ & $\begin{array}{l}\text { Diminuição da parvalbumina em córtex pré- } \\
\text { frontal. }\end{array}$ \\
\hline
\end{tabular}

neonatal em comparação aos controles, no qual foi aplicado solução salina no princípio da vida. Esta redução na produção de citocinas está associada à redução da fosforilação de $\mathrm{kkB}$ no fígado e baço, levando à diminuição da formação de NF-kB e consequente redução na ativação de prostaglandina E2 (catalisada pela COX-2) no cérebro. Esta linha de raciocínio conduz à possibilidade de que estas alterações possam ser devidas ao prejuízo no feedback negativo da corticosterona. Os glicocorticoides 
modificam a expressão de citocina, principalmente por suas ações sobre o NF-kB e eixo $\mathrm{HPA}^{33}$. Uma única aplicação de LPS durante o desenvolvimento impacta também no processamento sensorial e doloroso em indivíduos adultos, levando a diminuição dos limiares nociceptivos e aumento das respostas a estímulos dolorosos 24 .

Uma resposta febril competente é parte crucial de defesa do hospedeiro contra infecções, sendo essencial para a sobrevivência. A febre tem um papel importante na potencialização das respostas imunológicas e na alteração da temperatura ótima para o crescimento patogênico (diminuindo sua proliferação). Assim, a diminuição da resposta térmica após a infecção, como em casos de hipotermia na sepse, conduz à menor sobrevida. Spencer ${ }^{22}$ mostra que o desafio imunológico com a administração de LPS no período neonatal atenua a hipotermia e acentua a resposta febril em animais adultos submetidos a doses sépticas de LPS, mostrando uma resposta positiva de adaptação. Este dado conflita com outros trabalhos, que mostram atenuação da febre em situações similares ${ }^{33}$.

Ainda, ao analisarem-se efeitos comportamentais, sabe-se que o período neonatal é um estágio muito importante na compreensão da etiologia e da trajetória do aparecimento de desordens neuropsiquiátricas na vida adulta, como a ansiedade e a depressão ${ }^{12}$. Exposição neonatal de LPS em roedores está associada com o aumento do comportamento de ansiedade, independente da exposição estressora no adulto ${ }^{16}$. Ratos neonatos tratados com LPS mostram um marcado aumento na sensibilidade ao estresse quando adultos se comparados com controles tratados com salina, mostrando efeito de programming do eixo HPA em longo prazo como resposta ao estresse inicial. $O$ mecanismo pelo qual o LPS desenvolve este efeito não é completamente entendido, embora a administração de LPS mimetize os eventos ocorridos durante o estresse infeccioso, incluindo resposta febril, aumento da produção de citocinas como a IL-1 $\beta$, IL-6 e TNF- $\alpha$ pelo aumento na atividade da COX-2 e produção de PGE2 ${ }^{25}$. É bem estabelecido que o sistema imune é afetado pelo status neurológico do hospedeiro e também afeta a condição neurológica do indivíduo ${ }^{34}$, ou seja, há uma estreita interação entre os sistemas neuroendócrino e neuroimune, havendo comunicação bidirecional entre eles $^{29}$. Um problema no estudo das consequências comportamentais da doença é a dificuldade de separar os efeitos do agente infeccioso em si dos efeitos da resposta imunológica a este agente ${ }^{20}$.

De maneira geral, o estresse é uma resposta interna ao estímulo ou pressão que muda ou destrói a homeostasia do organismo em resposta a uma alteração ambiental ${ }^{1}$. O eixo HPA desempenha papel primordial na regulação da função metabólica. Seu efeito constitutivo é importante componente na resposta ao estresse ${ }^{1}$, de tal sorte que alterações neste eixo hormonal são frequentemente associadas a distúrbios de ansiedade e depressão, bem como à violência e agressão ${ }^{35}$. Animais expostos à endotoxina durante a primeira semana de vida são mais responsivos ao estresse quando adultos, mostrando aumento de hormônio adenocorticotrópico (ACTH) e corticosterona, além de diminuição de receptores de glicocorticoides no hipotálamo, córtex frontal e hipocampo ${ }^{28}$. Os glicocorticoides são os primeiros mediadores no programming do eixo HPA, sendo assim, seus receptores (responsáveis por seus efeitos) exercem papel fundamental na resposta ao estresse. Em camundongos transgênicos, a redução de 30-50\% nos níveis de receptores, resulta em significante desordem neuroendócrina, metabólica e imunológica ${ }^{1}$.

A doença comportamental é um estado de motivação desencadeada pela ativação do sistema imune inato periférico, caracterizada por redução na atividade locomotora nos comportamentos exploratórios ${ }^{36}$. Vários modelos de estresse precoce em roedores, incluindo o estresse pré-natal e pós-natal, têm sido desenvolvidos para o estudo dos mecanismos neurobiológicos de distúrbios relacionados com ansiedade e depressão. Geralmente, o estresse precoce em animais aumenta o comportamento de ansiedade e depressão, e é acompanhado por alterações funcionais do eixo HPA ${ }^{34}$. Quando adultos, ratos neonatais infectados com E. coli exibem certo número de alterações fisiológicas e comportamentais, incluindo a vulnerabilidade a deficiências cognitivas, redução da resposta da corticosterona ao estresse e diminuição das interações sociais $^{37}$, sendo este perfil particularmente aparente quando os animais são submetidos à um evento 
estressor agudo adicional quando adultos. Uma das observações mais consistentes é que a exposição ao LPS nos estágios iniciais de vida resulta em prole que, na idade adulta, demonstra aumento no comportamento de ansiedade e depressão. Ademais, evidência recente indica que estes resultados comportamentais não estão limitados aos animais expostos isoladamente, mas têm sido demonstrados ao persistirem numa geração subsequente de descendentes, carregados por uma linha materna ou paterna dos animais previamente expostos ao LPS $^{30}$.

Com isso, esta revisão demonstra os efeitos gerados em longo prazo pela administração neonatal de LPS. Este procedimento vem sendo utilizado largamente na literatura como modelo experimental para estudo dos efeitos do programming. Ratos submetidos ao desafio com LPS no início da vida apresentam alterações imunológicas e comportamentais na vida adulta. O "duplo desafio" imunológico sugere alterações funcionais do eixo HPA, o que propicia concentrações circulantes das citocinas pró-inflamatórias IL-1ß, IL-6 e TNF- $\alpha$ significativamente atenuadas, hiperalgesia, aumento na sensibilidade ao estresse e aumento do comportamento de ansiedade e depressão. Um melhor conhecimento destes modelos e seus efeitos, à luz da literatura atual, possibilita que se avance no entendimento dos mecanismos do programming, propiciando melhor manejo de seus efeitos.

\section{REFERÊNCIAS}

1. Xiong F, Zhang L. Role of the hypothalamic-pituitaryadrenal axis in developmental programming of health and disease. Front Neuroendocrinol. 2013;34(1):27-46.

2. Iwasa $T$, Matsuzaki T, Kinouchi R, Fujisawa $S$, Murakami M, Kiyokawa M, Kuwahara A, Yasui $T$, Irahara $M$. Neonatal LPS injection alters the body weight regulation systems of rats under non-stress and immune stress conditions. Int J Dev Neurosci 2010;28(1):119-24.

3. Padoin MJ, Cadore LP, Gomes CM, Barros HMT, Lucion $A B$. Long-lasting effects of neonatal stimulation on the behavior of rats. Behav Neurosci 2001;115:1332-40.

4. Levine S. The psychoendocrinology of stress. Ann NY Acad Science 1993;697:61-9.

5. Meaney MJ, Seema B, LaRocque S, McCormick C, Shanks N, Sharma S, Smythe J, Viau V, Plotsky PM. Individual differences in the hypothalamic-pituitary-adrenal stress response and the hypothalamic CRF system. Ann N Y Acad Sci. 1993;697:70-85.

6. Gomes CM, Frantz PJ, Sanvitto GL, Anselmo-Franci JA, Lucion $\mathrm{AB}$. Neonatal handling induces anovulatory estrous cycles in rats. Braz J Med Biol Res. 1999;32(10):1239-42.
7. Gomes CM, Raineki C, Paula PR, Severino GS, Helena CVV, Anselmo-Franci JA, Franci CR, Sanvitto GL, Lucion $A B$. Neonatal handling and reproductive function in female rats. J Endocrinol 2005;184:435-45.

8. Lucion AB, Pereira FM, Winkelmann EC, Sanvitto GL, Anselmo-Franci JA. Neonatal handling reduces the number of cells in the locus coeruleus of rats. Behav Neurosci. 2003;117(5):894-903.

9. Gomes CM, Donadio MV, Franskoviaki I, Anselmo-Franci JA, Franci CR, Lucion AB, Sanvitto GL. Neonatal handling reduces angiotensin $\|$ receptor density in the medial preoptic area and paraventricular nucleus but not in arcuate nucleus and locus coeruleus of female rats. Brain Research 2006;1067(1):177-80.

10. Donadio MV, Jacobs S, Corezola KL, Melo DA, Dias HB, Reichel CL, Franci CR, Jeckel-Neto EA, Lulhier F, Lucion $A B$, Oliveira JR, Sanvitto GL. Neonatal handling reduces renal function in adult rats. Kidney Blood Press Res 2009;32(4):286-92.

11. Karrow NA. Activation of the hypothalamic-pituitaryadrenal axis and autonomic nervous system during inflammation and altered programming of the neuroendocrine-immune axis during fetal and neonatal development: lessons learned from the model inflammagen, lipopolysaccharide. Brain Behav Immun 2006;20(2):144-58.

12. Amani $M$, Samadhi $H$, Doosti $M H$, Azarfarin $M$, Bakhtiari $A$, Zolbanin NM, Rahimi MM, Salari AA. Neonatal NMDA receptor blockade alters anxiety- and depression-related behaviors in a sex-dependent manner in mice. Neuropharmacology 2013;73:87-97.

13. Benatti C, Alboni S, Capone G, Corsini D, Caggia F, Brunello N, Tascedda F, Blom JM. Early neonatal inflammation affects adult pain reactivity and anxiety related traits in mice: genetic background counts. Int J Dev Neurosci 2009;27(7):661-8.

14. Li XF, Jones JSK, Knox AMI, Wu XQ, Tahsinsoy D, Brain SD, Lightman SL, O'Byrne KT. Neonatal lipopolysaccharide exposure exacerbates stress-induced suppression of luteinizing hormone pulse frequency in adulthood. Endocrinology 2007;148:5984-90.

15. Jenkins TA, Harte MK, Stenson G, Reynolds GP. Neonatal lipopolysaccharide induces patological changes in parvalbumin immunoreactivity in the hippocampus of the rat. Behav Brain Res. 2009;205(2):355-9.

16. Walker AK, Nakamura T, Byrne RJ, Naicker S, Tynan RJ, Hunter M, Hodgson DM. Neonatal lipopolysaccharide and adult stress exposure predisposes rats to anxiety-like behaviour and blunted corticosterone responses: implications for the double-hit hypothesis. Psychoneuroendocrinology 2009;34(10):1515-25.

17. Iwasa $T$, Matsuzaki $T$, Murakami M, Kinouchi R, Ogata R, Kuwahara A, Yasui T, Irahara M. Neonatal lipopolysaccharide exposure attenuates the homotypic stress-induced suppression of $\mathrm{LH}$ secretion in adulthood in male rat. Int J Dev Neurosci. 2009;27(4):345-9.

18. Gilmer WS, McKinney WT. Early experience and depressive disorders: human and non-human primate studies. J Affect Disord. 2003;75(2):97-113.

19. Gil AC. Como elaborar projetos de pesquisa. $4^{\text {a }}$ ed. São Paulo: Atlas; 2006.

20. Hood KE, Dreschel NA, Granger DA. Maternal behavior changes after immune challenge of neonates with developmental effects on adult social behavior. Dev Psychobiol 2003;42(1):17-34.

21. Iwasa $T$, Matsuzaki T, Murakami M, Kinouchi R, Shimizu F, Kuwahara A, Yasui T, Irahara M. Neonatal immune challenge affects the regulation of estrus cyclicity and feeding behavior in female rats. Int $\mathrm{J}$ Dev Neurosci 2009;27(1):111-4. 
22. Spencer SJ, Field E, Pittman QJ. Neonatal programming by neuroimmune challenge: effects on responses and tolerance to septic doses of lipopolysaccharide in adult male and female rats. J Neuroendocrinol 2010;22(4):27281.

23. Kentner AC, McLeod SA, Field EF, Pittman QJ. Sexdependent effects of neonatal inflammation on adult inflammatory markers and behavior. Neuroendocrinology 2010;151:2689-99.

24. Boissé L, Spencera SJ, Mouihatea A, Vergnolleb N, Pittman QJ. Neonatal immune challenge alters nociception in the adult rat. Pain;119(1-3):133-41.

25. Knox AM, Li XF, Kinsey-Jones JS, Wilkinson ES, Wu XQ, Cheng YS, Milligan SR, Lightman SL, O'Byrne KT. Neonatal lipopolysaccharide exposure delays puberty and alters hypothalamic Kiss1 and Kiss1r mRNA expression in the female rat. J Neuroendocrinol 2009;21(8):683-9.

26. Tenk CM, Foley KA, Kavaliers M, Ossenkopp KP. Neonatal immune system activation with lipopolysaccharide enhances behavioural sensitization to the dopamine agonist, quinpirole, in adult female but not male rats. Brain Behav Immun 2007;21(7):935-45.

27. Li XF, Kinsey-Jones JS, Knox AM, Wu XQ, Tahsinsoy D, Brain SD, Lightman SL, O'Byrne KT. Neonatal lipopolysaccharide exposure exacerbates stress-induced suppression of luteinizing hormone pulse frequency in adulthood. Endocrinology 2007;148(12):5984-90.

28. Shanks N, Windle RJ, Perks PA, Harbuz MS, Jessop DS, Ingram CD, Lightman SL. Early-life exposure to endotoxin alters hypothalamic-pituitary-adrenal function and predisposition to inflammation. Proc Natl Acad Sci USA 2000;97(10):5645-50.

29. Walker AK, Nakamura T, Hodgson DM. Neonatal lipopolysaccharide exposure alters central cytokine responses to stress in adulthood in Wistar rats. Stress 2010;13(6):506-15.

30. Sominski L, Fuller EA, Bondarenko E, Ong LK, Averell L, Nalivaiko E, Dunkley PR, Dickson PW, Hodgson DM.
Functional programming of the autonomic nervous system by early life immune exposure: implications for anxiety. Plos One 2013;8(3):1-13.

31. Walker FR, Hodyl NA, Krivanek KM, Hodgson DM. Early life host-bacteria relations and development: long-term individual differences in neuroimmune function following neonatal endotoxin challenge. Physiol Behav. 2006;87(1):126-34

32. Jenkins TA, Harte MK, Stenson G, Reynolds GP. Neonatal lipopolysaccharide induces pathological changes in parvalbumin immunoreactivity in the hippocampus of the rat. Behav Brain Res 2009;205(2):355-9.

33. Spencer SJ, Galic MA, Pittman QJ. Neonatal programming of innate immune function. Am J Physiol Endocrinol Metab 2011;300:E11-E18.

34. Kumar V, Sharma A. Is neuroimmunomodulation a future therapeutic approach for sepsis? Int Immunopharmacol 2010;10(1):9-17.

35. Neumann ID, Veenema AH, Beiderbeck DI. Aggression and anxiety: social context and neurobiological links. Front Behav Neurosci 2010;4:1-12.

36. Frolkis I, Klein Y, Locker C, Adi N, Dahan E, Uretzsky G, Shapira I, Sorkine P. Vipera aspis venom reduces lethality and down-regulates tumor necrosis factor-alpha in a rat model of LPS-induced sepsis. Cytokine 2010;49(3):31924.

37. Bilbo SD, Schwarz JM. The immune system and developmental programming of brain and behavior. Front Neuroendocrinol. 2012;33(3):267-86.

\section{Endereço para correspondência}

Márcio Vinícius F. Donadio

Av. Ipiranga $n^{\circ} 6690,2^{\circ}$ andar (IPB)

Porto Alegre/RS - CEP 90610-000

Telefone: +55 5133203500 (r. 2313)

E-mail: mdonadio@pucrs.br 